\title{
Luft sehen, sprechen, schützen
}

Das Anthropozän der (post-)politischen Stadt

\author{
Friederike Landau \\ Alexandra Toland
}

\begin{abstract}
Der Beitrag verbindet die Diskussion um die postpolitische Stadt mit der zunehmenden wissenschaftlichen und aktivistischen Auseinandersetzung mit dem Anthropozän, ein Konzept, das die ökologischen und sozialpolitischen Implikationen menschlichen Handelns auf die Erdoberfläche beschreibt. Anhand von drei ausgewählten Fallstudien erkunden wir, wie die spezifisch anthropogene, also menschengemachte, Krise urbaner Luftverschmutzung in künstlerischen Positionen problematisiert wird. Im Kontext des potenziellen Vormarschs von Postpolitik besprechen wir, wie der ambivalente Diskurs des Anthropozäns einerseits Depolitisierung begünstigt und andererseits neue Möglichkeiten für die Repolitisierung globaler Umweltherausforderungen ermöglicht.
\end{abstract}

Ersteinreichung: 28. März 2019; Veröffentlichung online: 24. April 2020

An English abstract can be found at the end of the document.

\section{Anthropozän als (Post-)Politikum}

Ein Mann mit Pferdeschwanz, Mitte 30, läuft im Abendlicht durch die Straßen von Beijing. Er hält einen handelsüblichen Staubsaugerschlauch in der Hand, mit ernster Miene, aber auch ein bisschen triumphierend. Hinter sich zieht er einen selbstgebauten Wagen, auf dem der Staubsauger thront. Das Gesicht hinter einer Atemmaske verborgen, saugt der Mann Smogpartikel auf, die täglich in Städten umherschwirren und sich auf Gegenständen, Oberflächen, Haut und in den Lungen ablagern. Der Kampf, den der chinesische Konzeptkünstler Nut Brother 2015 mit seiner Aktion „Project Dust“ anstößt, scheint kaum zu gewinnen zu sein - Mensch gegen menschengemachte Auswüchse industrieller Produktion, Ressourcenausbeutung, immer weiter fortschreitende Urbanisierung. In Nut Brothers Performance wird mit mikropolitischen Gesten eine symbolische Provokation zum Kontrollverlust des Menschen über den Planeten gezeigt. Er weckt mit seiner Kunst gleichzeitig Hoffnung und Hoffnungslosigkeit, was die Zukunft urbaner Lebensräume angeht.

Im folgenden Beitrag bringen wir die zunehmende wissenschaftliche und aktivistische Auseinandersetzung mit dem urbanen Anthropozän[1] (Derickson/MacKinnon 2015; Estok/Chou 2017; Karaliotas/Bettini 2016) mit der Diskussion um die postpolitische Stadt zusammen (Landau/Roskamm 2018; Michel/Roskamm 2013; Swyngedouw 2009). Anhand von drei 
ausgewählten Fallstudien erkunden wir, wie die spezifisch anthropogene Krise urbaner Luftverschmutzung in künstlerischen Positionen problematisiert wird. Wir untersuchen die künstlerisch-gestalterischen Praktiken der Vermittlung und Kommunikation von Umweltherausforderungen im Hinblick auf das Potenzial, neue Formationen des Politischen zu eröffnen. Hierbei konzentrieren wir uns insbesondere auf die von den aktivistischen Künstler*innen angestrebten affektiven und sensorischen Zugänge, wie in der oben beschriebenen Arbeit, um Ausmaß und Konsequenzen menschengemachter Luftverschmutzung erfahrbar zu machen. Wir schließen an bestehende Forschungen über die Rolle von Künstler*innen und Aktivist*innen im Kontext von Umweltgerechtigkeit an (Cauter et al. 2011; Landau/Toland im. Ersch.; McKee 2017) und leisten einen Beitrag zum Verständnis, wie Künstler*innen und Designer*innen sich im oder vielmehr gegen das Anthropozän positionieren (Davis/Turpin 2015; Demos 2017). Wir begreifen das Anthropozän als ambivalenten Diskurs, der einerseits Depolitisierung begünstigt und andererseits neue Möglichkeiten für die Repolitisierung von globalen Umweltherausforderungen eröffnet.

Wir diskutieren die Gesellschaftsdiagnosen des Anthropozäns und der Postpolitik, also des Verschwindens, des Endes oder des Tods der Politik (Swyngedouw 2009), als ineinander verschränkte Diskurse, die Praktiken und Bewusstsein für Umwelthandeln und -aktivismus beeinflussen. Entgegen der Annahme, dass sich postpolitische Regierungstechniken nahezu unaufhaltsam ausbreiten, verstehen wir die Postpolitik-Diagnose nicht als apokalyptisches Ende einer Diskussion, sondern als Ausgangspunkt der Erkundung, was an ebendieser Debatte politisch sein könnte oder sollte. Unser Ziel ist es, den Diskurs über Postpolitik zu (re-)politisieren (Jessop 2014). Der Diskussion der politisierenden Effekte von künstlerischem Umweltaktivismus liegt ein Verständnis von Politik zugrunde, welches auf der Differenz zwischen ,Politik' und ,dem Politischen' aufbaut (Marchart 2010; Mouffe 2005). Im engeren Sinne verstehen wir Politik als institutionalisierte und routinisierte Verfahrens- und Verhaltensweisen. Im postpolitischen Kontext läuft die Politik Gefahr, „das offene, störende, nicht festgelegte und verändernde Moment abzuschaffen" (Michel/Roskamm 2013: 9; Swyngedouw 2011). Im postpolitischen Regime wird das Politische unterdrückt oder ausgeschlossen, obwohl die Widerspenstigkeit und Konflikthaftigkeit des Politischen eigentlich unauslöschlich sind. In anderen Worten: Das Politische, oder Dimensionen von Konflikt, Antagonismus oder Agonismus, sind grundlegend und notwendig für die Konstitution von Objekten wie Gesellschaft, Politik oder Natur und werden durch die Beschränkung technokratischer Management-Rationalitäten auf ein Auswählen zwischen mehr oder weniger gleichen Optionen verschleiert (Marchart 2013).

Im Folgenden untersuchen wir künstlerische Positionen im Hinblick auf ihre vielschichtigen Möglichkeiten, neue Formationen zwischen Politik und dem Politischen zu artikulieren, oder das Politische durch künstlerische Aktionen zu repolitisieren. In Bezug zum kontroversen Diskurs des Anthropozäns wollen wir aufzeigen, dass dieser Diskurs Depolitisierung nicht notwendigerweise vertieft (Swyngedouw/Ernstson 2018), sondern dass trotz - und gerade wegen - der Ambivalenz und Mehrdeutigkeit des AnthropozänDiskurses Möglichkeiten erschlossen werden können, um das Politische neu 
zu denken (Kaika/Swyngedouw 2014). In der spezifischen Verknüpfung der angeblich postpolitischen Stadt mit anthropogenen Auswirkungen auf deren ,Natur hinterfragen wir einerseits die Dominanz unternehmerischer, meist neoliberaler Ansätze städtischer Steuerung sowie die menschengemachten Unterscheidungen zwischen Natur und Kultur/Stadt(Demos 2009).Zugleich diskutieren wir künstlerische Interventionen als (Ver-)Handlungsräume für die Problematisierung und Affizierung sowie auch die Entwicklung von Lösungsansätzen gegen den sich planetarisch ausbreitenden Zustand anthropogener Umweltzerstörung (Ruddick 2015).

\section{Kunst gegen das Anthropozän - affektive Assemblagen}

Wir betrachten Kunst und Politik sowie das Politische nicht als voneinander getrennte Sphären, die sich separat behandeln oder erfahren lassen könnten, sondern verstehen sie als unausweichlich miteinander verbundene Bereiche von Bedeutungen, Gefühlen und Macht (Mouffe 2005). Ohne Kunst per se emanzipatorische Kraft zuzuschreiben, begreifen wir künstlerische Produktion als grundlegend politisch und antagonistisch. Auch wenn das Verhältnis zwischen Kunst und Aktivismus in der zeitgenössischen Kunstgeschichte (Bishop 2010; Kester 1998; Scott 2014), Kultursoziologie (Hieber/ Möbius 2011), Kulturgeographie (Buser et al. 2013), urbaner Ethnographie (Juris/Khasnabish 2013), sozialer Bewegungsforschung (Hollands/Vail 2012) und Kulturpolitik (Landau 2019; Woddis 2005) kontrovers diskutiert bleibt, entfaltet der kursorische Überblick von drei Beispielen die Bandbreite des künstlerisch-aktivistischen Engagements im oder gegen das Anthropozän.

Seit 2013 beschäftigt sich das Berliner Haus der Kulturen der Welt (HKW) mit dem Anthropozän in unzähligen Ausstellungen, Panel-Diskussionen, Film- und Performance-Projekten[2]; auch verschiedene Kinofilme[3] und Museumsausstellungen[4] thematisierten in letzter Zeit das Anthropozän mehr oder weniger explizit in der Kunstwelt. Heather Davis und Etienne Turpins Sammelband Art in the Anthropocene - Encounters Among Aesthetics, Politics, Environments and Epistemologies (2015) sowie die vom HKW herausgegebene dreibändige Reihe namens Textures of the Anthropocene: Grain Vapor Ray (Klingan et al. 2014) bieten bis dato transdisziplinäre, eklektische Essay-Sammlungen, die sowohl zeitgenössische künstlerische Projekte über das Anthropozän vorstellen als auch den gesamtgesellschaftlichen Diskurs in eine reflexive Diskussion von Kunstund Architekturtheorie und -praxis einbetten. Die Potenziale künstlerischer Medien wurden auch im Kontext von Klimaforschung bereits besprochen (Klöckner et al. 2014; Renn/Scherer 2017). Jürgen Renn, Direktor am MaxPlanck-Institut für Wissenschaftsgeschichte, hält den Begriff des Anthropozäns für hilfreich, da er „unser Verständnis des Verhältnisses von Kultur und Natur in eine Blickperspektive bringt, und dass wir uns [hier] auf geologische Erkenntnisse beziehen können, das gibt auch der menschlichen Geschichte eine neue Dimension, denn hier verwebt sich die menschliche Geschichte mit der Erdgeschichte“ (Renn/Karkowsky 2018). Im Sinne dieser Möglichkeiten, neue Dimensionen von Geschichte und Gegenwart zu erfahren, auch sinnlich zu erfahren, diskutieren wir künstlerischen Aktivismus gegen das Anthropozän als politisierende Praxis. 
Im Wissen um die Kritik der potenziellen Ästhetisierung des AnthropozänDiskurses durch Kunst (Falb 2015; Rowan 2015) geht es uns nicht vorrangig um eine Besprechung spektakularisierender, Distanz einnehmender, rein visueller Repräsentationen des Anthropozäns (Demos 2017; Falb 2015). Stattdessen fokussieren wir uns auf interdisziplinäre, multi-mediale und multi-sensorische künstlerische und gestalterische Positionen, die das Anthropozän als Ansammlung oder Assemblage heterogener materieller, diskursiver und affektiver Infrastrukturen und Atmosphären be- und aufgreifen (Bignall 2010; Reestorff 2015; Street 2012).[5] Uns geht es in der Analyse der Fallstudien um die Erzeugung und das Sicht- und Fühlbarmachen von Hoffnungen, Gefühlen, Bildern und Imaginationen als Ausformungen des Politischen und der Anthropozän-Assemblage, die die künstlerischen und gestalterischen Interventionen hervorrufen können. Kathryn Yusoff und Jennifer Gabrys verstehen Imaginationen als vielschichtige „Wege des Sehens, Fühlens, Denkens und Träumens der Formation von Wissen, welche Bedingungen materieller Interventionen in und politische Sensibilitäten von der Welt erschaffen werden können“ (2011: 516, Übers. d. A., Herv. im Orig.). Ihrem Verständnis folgend, begreifen wir politische Subjekte und Objekte, die neueZukunftsvisionen aufbauen, als epistemologisch und affektiv miteinander verwoben. Das heißt, neue Vorstellungen von und über mögliche und wünschenswerte Zukünfte entstehen, indem Beziehungen und Bedeutungen von Mensch, Umwelt, Natur, Kultur und Stadt radikal neu geordnet werden.

Im Zuge des ,affektiven Turns ' in den Geistes-, Kultur- und Sozialwissenschaften gewinnen körperliche, sensorische und emotionale Dimensionen für Macht- und Gesellschaftsanalysen an Bedeutung (Gregg/Seigworth 2011). Wir verstehen Affekte als trans-individuelle und materiell-diskursive Kräfte und, in Anlehnung an Spinoza, als Fähigkeiten von Körpern, Macht oder Aktivität anderer Körper zu erhöhen oder zu verringern. Emotionen können als individuelle oder persönlich gefühlte Wahrnehmungen verstanden werden.[6] Es ist für uns weniger relevant, welche Art von Affekten oder Emotionen (z. B. Angst, Scham, Freude, Empathie, Hoffnung, Panik etc.) konkret hervorgerufen werden, oder ob diese Affizierung ,effektiv' ist (wie auch immer mensch das messen könnte), sondern darum, Implikationen des Affizierens und Affiziertwerdens durch das Anthropozän sowohl in affektiven Assemblagen als auch in künstlerisch-gestalterischen Interventionen zu identifizieren (siehe Mohr/Landau 2017).[7]

Die affektive Assemblage dient als methodologische Orientierung für die Beachtung menschlicher, nicht-menschlicher, materieller, diskursiver, technischer und körperlicher Bestandteile vielstimmiger Anordnungen, die variable Fähigkeiten aufweisen, zu affizieren und affiziert zu werden. Reestorff (2015: 202) hebt hervor, dass sowohl die Komponenten als auch die Assemblage als Ganze sich durch verschiedene Kapazitäten von ,agency verändern können. Reestorff definiert Assemblage-Bestandteile in Anlehnung an Manuel Castells und Manuel de Landa als

„[...] sowohl materiell als auch rein expressiv. In einer Assemblage spielen sinnliche Eindrücke eine wichtige Rolle und nicht nur visuelle, aurale, olfaktorische und taktile, sondern auch die Vielfalt der 
Leidenschaften, von Stolz und Erniedrigung bis zu Liebe und Hass, besitzen ihre eigene singuläre Individualität.“(ebd.: 210, Übers. d. A.)

Der Assemblage-Ansatz ermöglicht eine Betrachtung von künstlerischen Aktionen über das Kunstobjekt, -produkt oder -ding hinaus und markiert den Kunstgegenstand nicht als alleinigen Auslöser der Affizierung durch ,die' Kunst. Stattdessen steht die Ausstrahlung oder Transmission affektiver Atmosphären im Vordergrund, die ästhetische Objekte oder künstlerische Interventionen als prozessuale, politische und vernetzte Materialitäten konstituieren kann (Anderson 2009: 79).

\section{Public Smog - das noch ungefühlte Anthropozän}

Die amerikanische Künstlerin Amy Balkin wendet sich in ihrem Projekt „Public Smog“[8] (2004 bis heute) der Idee eines Saubere-Luft-Parks zu (clean air park). Die Aktion hat keinen konkreten Anfang, kein Ende und keinen Performance-Charakter, sondern besteht im Wesentlichen aus der Ankündigung der Eröffnung und dem theoretischen Erfahren sauberer(er) Luft. Zunächst rief Balkin 2004 ein solches Konstrukt im kalifornischen South Coast Air Quality Management District aus und weitete die Zonen anschließend auf die Europäische Union (2007) und Kamerun (2009) aus. Zur Eröffnung der Saubere-Luft-Parks erwarb Balkin in international regulierten Emissionsmärkten Ausgleichspapiere, um diese Emissionsrechte nicht auszunutzen, sondern im übertragenen Sinne als saubere Luft der Allgemeinheit zur Verfügung zu stellen. Balkin macht durch den kommerziellen Akt des Entzugs von Anteilsscheinen auf die umweltschädlichen Industrien aufmerksam, die Luft auf dem Wege des Lufthandels durch ihre Produktion legal und mit Erlaubnis verpesten (Balkin 2012; Yusoff/Gabrys 2011). Durch den Kauf und die Nichtinanspruchnahme von Emissionsscheinen, also die Inversion der eigentlichen Funktion der Emissionspapiere, eröffnet Balkin einen gedanklich-politischen und quasi-physischen Raum und den Diskurs um ein Recht auf saubere Luft, um die Kommodifizierung von Luft zu kritisieren (vgl. Abb. 1). Ihre Motivation für das Projekt beschreibt Balkin folgendermaßen:

„Für mich war dieser Emissionshandel eine Art von Landnahme (land grabbing) in der Atmosphäre. Das war es, was mich motiviert hat; nicht nur, diese Landnahme zu repräsentieren, sondern auch sie zu verstehen und zu intervenieren, wenn auch auf einem sehr geringen Level. Ich wollte verstehen, wie das funktioniert, und dann die gleichen Mechanismen verwenden, von denen ich dachte, dass sie etwas zum Zuspitzen (der Situation) beitragen.“ (AB 2018[9])

Der angesprochene Widerstand gegen die Landnahme, oder eher ,Luftnahme', spiegelt Balkins Verständnis von Luft als öffentlichem Luftraum oder gemeinschaftlichem Gut wider. Die Anspielung auf die Verwendung ,gleicher Mechanismen“ wie die des internationalen Luftemissionshandels verweist auf die Herausforderung der Grenzen zwischen Politik und dem Politischen beziehungsweise deren Re-Interpretation. Balkin kollektiviert die Eigentümer*innenschaft guter Luft und politisiert so den Kauf oder 
die Käuflichkeit von Luft, die eigentlich Eigentum von ,allen“ ist oder sein sollte. Einerseits appelliert Balkin so an ,alle' als gemeinschaftlich betroffene Subjekte von Luftverschmutzung. Statt einer Totalisierung von ,allen` zeigt Balkins Projekt jedoch andererseits auch die stark ungleiche Verteilung der Verursachung sowie die asymmetrische Betroffenheit von den Konsequenzen des Anthropozäns auf (siehe Derickson/MacKinnon 2015). Damit schließt sich Balkin wiederum der queer-feministischen Kritik am Konzept des Anthropozäns an, welches, den Menschen' oft als universales Subjekt behandelt und unterschiedlich gelagerte Ausbeutungs- und Leidensverhältnisse im Hinblick auf Geschlecht, Alter, Sexualität, Ethnizität und sozioökonomisches Kapital vereinheitlicht (Davis/Todd 2017; Haraway 2016), sodass man bei ihrem Projekt auch von einer Kritik am Kapitalozän (Haraway/Kenney 2015; Moore 2016) sprechen könnte. Balkin zeigt, dass luftverschmutzende Industrien wie Öl- und Kohleextraktion oder bleiverarbeitende Gewerbe einen wesentlich höheren Anteil an der Verschmutzung haben als individuelle Nutzer*innen der Atmosphäre. Auch wenn die von Balkin erworbenen Emissionsscheine einem David*-gegen-Goliath*Verhältnis ähneln, gelingt der Künstlerin ein Eingriff in den kommerziellen Handel mit Luftemissionen. Dieser fordert durch die verkehrte Nutzung ihrer erworbenen Rechte zur Luftverschmutzung die institutionalisierten Strukturen der Verschmutzungspolitik heraus. Balkin agiert zwar als Individuum, handelt und appelliert aber an eine abstrakte Öffentlichkeit, der die Künstlerin ihre Saubere-Luft-Parks zur Verfügung stellen möchte. Dennoch reicht Balkins Appell und auch ihr eigenes Engagement weit über einen symbolischen Akt hinaus; die Projekt-Website von „Public Smog“ versammelt verschiedene internationale aktivistische Vereinigungen, die sich mit künstlerischen oder legalen Mitteln gegen die Kommodifizierung des Luftraums engagieren.

Die feldübergreifende Reichweite und internationale Rezeption der Initiative wurden 2012 noch verdeutlicht, als Balkin als Teilnehmerin der documenta 13 eingeladen wurde. In einer öffentlichkeitswirksamen Aktion versuchte sie, die Erdatmosphäre als (immaterielles) Weltkulturerbe zu etablieren. Hierzu rief sie Besucher*innen der documenta 13 auf, vorgefertigte Postkarten zu unterzeichnen, die in drängendem Duktus auf die Notwendigkeit hinweisen, die Erdatmosphäre nachhaltig zu schützen. Balkin präsentiert einen Auszug der Postkarte, „dem öffentlichen Interesse folgend, die Atmosphäre für gegenwärtige und zukünftige Generationen zu schützen, und in Anerkennung, dass ihre Bewahrung die Pflicht der internationalen Gemeinschaft ist“ (2015: 341).

Im Zuge der documenta-Aktion versandte Balkin über 50.00o Postkarten an den damaligen deutschen Bundesumweltminister Peter Altmaier (CDU), der sich des Anliegens jedoch nicht annahm. 186 weitere Staatsoberhäupter von Mitgliedsstaaten der Vereinten Nationen wurden kontaktiert - außer von der Bildungs-, Frauen und Kulturministerin des Königreichs Tonga, einem südpazifischen Inselstaat, der stark von anthropogenen Klimaproblemen betroffen ist, erhielt die Künstlerin jedoch keine Antwort von Politiker*innen (Balkin 2015: 345).

Die Assemblage von „Public Smog“ besteht sowohl aus materiellen als auch diskursiven Bestandteilen: Das Projekt umfasst die bürokratischen, 
Abb. 1 Clean-AirPark (Quelle: Amy Balkin[10])

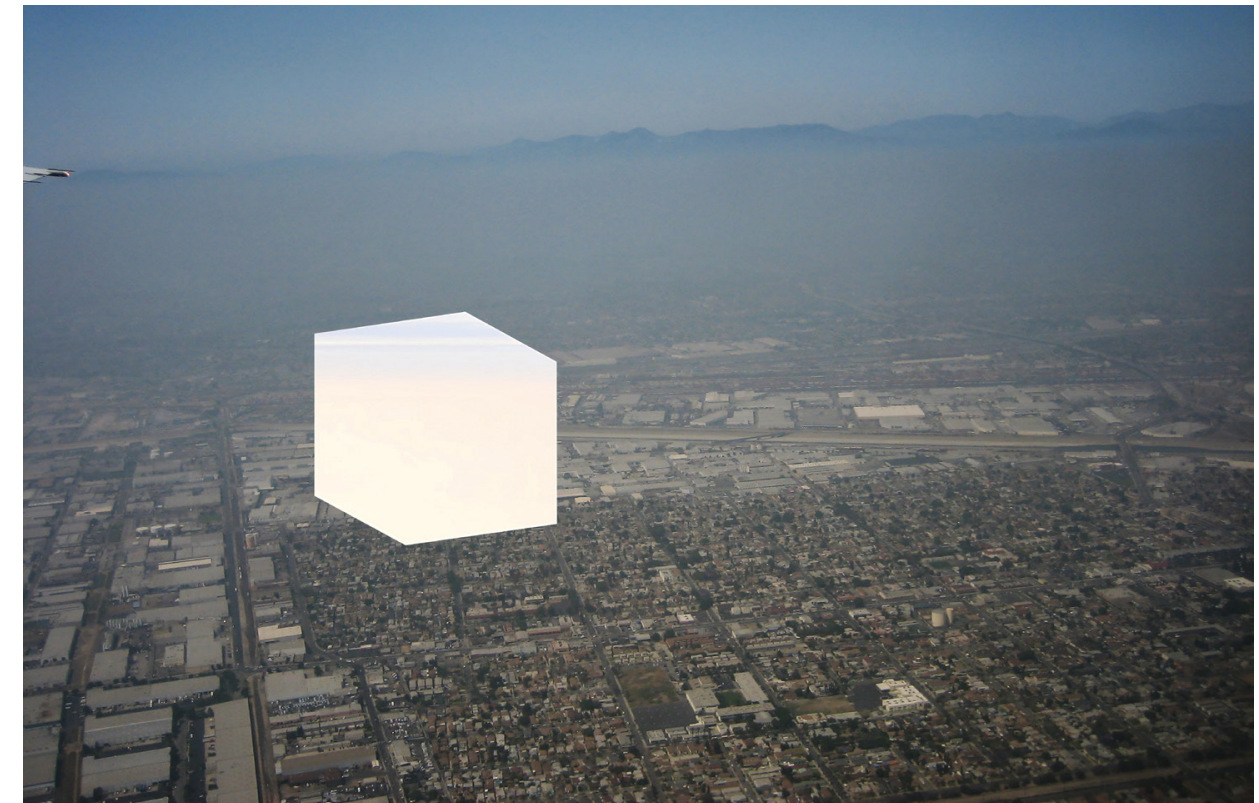

legalen und finanziellen Tätigkeiten des Emissionsanteilserwerbs, die fiktiven Bildbearbeitungen, die die Größe des Areals gekaufter sauberer Luft anzeigen sollen (vgl. Abb. 1), eine Plakatkampagne im öffentlichen Raum in Kamerun sowie die stetig wachsende technische Infrastruktur der Projekt-Website und Balkins fortdauerndes Engagement, über das Projekt zu sprechen und zu schreiben. Am wenigstens greifbar bleiben wohl die Eindrücke und Reaktionen, die beim Publikum erzeugt werden. Anders ausgedrückt, sind vielleicht die Eindrücke, die beim Publikum nichts Konkretes erzeugen, am eindrücklichsten. Nicht nur die Frage, wen oder was das Publikum des Projekts eigentlich umfasst, erweist sich als kompliziert (die Vögel, die über den weniger verschmutzten Luftraum fliegen? Anwohner*innen unter dem gedachten weißen Kubus sauberer Luft in Kalifornien oder der EU? Wo genau ist die saubere Luft zu spüren, zu schmecken, zu riechen?), sondern auch die Frage, wie, wo, wann und in welchem Kontext dieses diffuse Publikum von dem mehrteiligen Projekt erfährt.

Ob im Ausstellungskontext der documenta 13, online oder durch ein Plakat am Straßenrand in den ausgewählten Städten oder fernab davon, Balkins Saubere-Luft-Parks evozieren die theoretisch körperlich erfahrbaren Vorteile sauberer Luft, ohne sie tatsächlich jedoch körperlich erfahrbar zu machen oder einzulösen. Obwohl Balkin umgerechnet Emissionsrechte für etwa 24 Kilogramm potenziell zu verschmutzende oder aber als sauber zu erhaltende Luft erwarb, bleibt der direkte sinnliche Reiz beziehungsweise die Affizierung in Suspension. Wichtiger erscheint es, einen Wunsch, eine Sehnsucht, ein Bedürfnis, ein Verlangen oder die schiere Vorstellung von besserer Luft überhaupt zu aktivieren. Anders gesagt: Die konkrete Intervention in den Bereich der Emissionspolitik macht die Politik als unvollständigen Bereich des umkämpften Feldes des Politischen sichtbar und konfrontiert die politisch voneinander abgegrenzten Sphären von emissionshandelnden Expert*innen und zivilgesellschaftlichen (künstlerischen) Aktivist*innen miteinander. Auf sinnlicher, körperlicher Ebene spürt man das zurückgehaltene Luftvolumen vielleicht nicht, die Aktion ruft aber eventuell dazu auf, es spüren zu wollen. Mit dem nun bereits 15 Jahre andauernden „Public-Smog“-Projekt spannt Balkin ein Terrain des Politischen auf, das mehr umfasst als nur 
technokratische Emissionshandelspolitik, und bringt so die Vorherrschaft der Politik ins Wanken. Was nun? Darauf gibt Balkins Initiative keine konkrete Antwort. Das weitgefasste Publikum bleibt gegebenenfalls aktiviert und latent affiziert zurück. Für zukünftige Aktionen, wie beispielsweise das Wiederaufleben der Kampagne zur Eintragung der Erdatmosphäre in das Weltkulturerbe-Register, muss sich die Gemeinschaft des Politischen wieder neu formieren und zusammenschließen.

Balkins visualisierte, textuelle, gedankliche Intervention in Form der Ausrufung Sauberer-Luft-Parks versucht, die Dringlichkeit der ,unsichtbaren“ Problematik von Abgasbelastung auf lokalpolitische und globale Agenden zu setzen, und problematisiert performativ die Präsenz unsauberer Luft. Die Aktion zögert eine mögliche Affizierung durch tatsächlich geatmete bessere Luft hinaus, und weckt durch ebendieses Hinauszögern Bedürfnisse nach dieser besseren Luft. Durch Unterwanderung des dominanten Systems globaler Umweltpolitik, welches im internationalen Emissionshandel institutionalisiert ist, destabilisiert Balkin flüchtig die dominante Politik und fordert die Rückkehr des Politischen mit einer affektiven Utopie ein. Für die Annäherung an diese vielleicht nicht allzu utopische Utopie sucht Balkin Mitstreiter*innen, die sie durch ihr Projekt auf verschiedenen Wegen anspricht. Die affektive Utopie ist rückgebunden an eine gegenseitige Informierung von Wissenschaft, Politik und Kunst, und kann eine neue „Poesie von Wissensproduktion“ (AB 2018) vorantreiben. Diese Poesie verknüpft quantitative, evidenzbasierte Kriterien der Wissensvermittlung über Luftverschmutzung mit affektiven Zugängen oder Vorstellungen über die Zukunft. Balkin betreibt futuring', ein Anreißen, Machen, Vorstellen anderer Zukünfte (Yusoff/Gaybrs 2011). So entsteht eine daten- und faktengestützte sowie affektive Assemblage, um Auswirkungen des Anthropozäns nicht nur kennen-, sondern auch (fast) fühlen zu lernen. Zudem destabilisiert Balkin den globalen Nachhaltigkeitskonsens, der mithilfe apokalyptischer Ökologien der Angst (Davis 1999) kaum Alternativen zum Emissionshandel bietet. Balkins Irritation des bestehenden Systems legt die konfliktreichen Ursprünge der Kommodifizierung von Luft(-verschmutzung) als handelbare Ware frei. Sie bringt das Politische gerade durch diese Offenlegung des Antagonismus zwischen guter Luft und dessen Konstruktion als von Einzelinteressen gehandelte, verkäufliche Materie zum Vorschein. Schwelt der Konflikt (wieder) an der Oberfläche, im Alltag, in den Köpfen der Menschen, ist die Postpolitik zugunsten des Politischen zumindest zeitweise verdrängt.

\section{Project Dust - das steinharte Anthropozän}

100 Tage lang sammelte der chinesische Performance-Künstler Nut Brother in „Project Dust“ im Jahre 2015 Feinstaubpartikel auf Beijings Straßen mit einem handelsüblichen Staubsauger auf (vgl. Abb. 2). Anschließend goss er die Smogpartikel unter Hinzugabe von Lehm zu einem Backstein zusammen. Die unleugbare Präsenz und Materialität von Smog in der Form eines Backsteins (vgl. Abb. 3) verdeutlicht Nut Brother mit fast banalen Ausdrucksmitteln wie einem gewöhnlichen Haushaltsstaubsauger. Mögen auch geringe Ressourcen zur Umsetzung des Projekts ein Grund für die technisch simple Ausstattung von „Project Dust“ sein, liegt in dieser kongenialen 

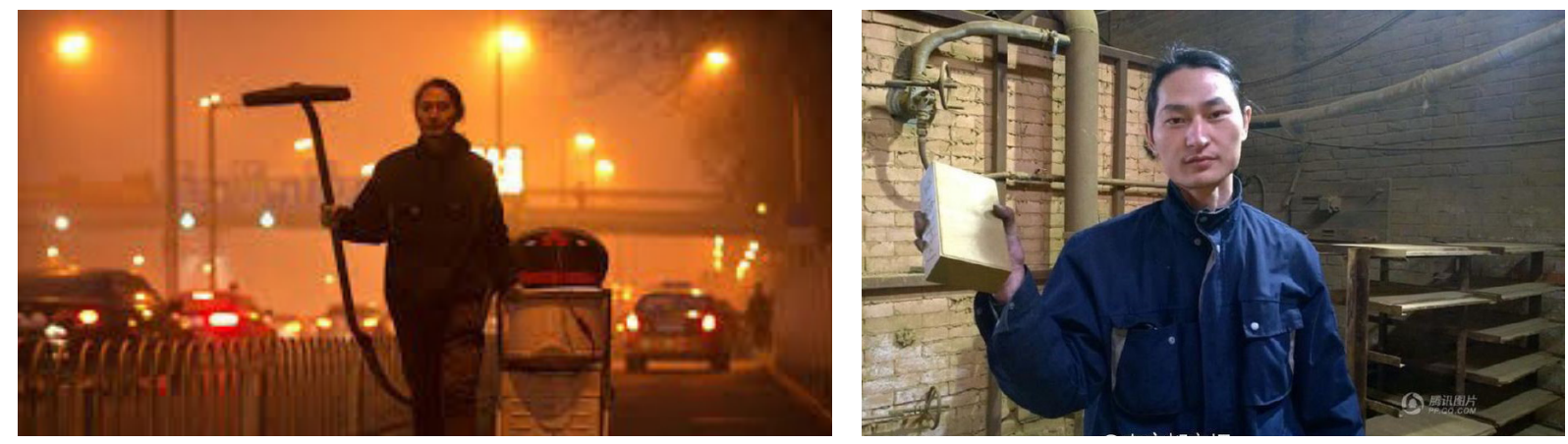

Abb. 2 Project Dust (Quelle: Berke 2015)

Abb. 3 Project Dust (Quelle: Ho 2015)
Vorgehensweise jedoch auch eine Ironie, die zur Problematisierung und Bekämpfung anthropogener Umweltzerstörung beiträgt. Im Gegensatz zu Verfechter*innen der These des „guten Anthropozäns“ (Bennett et al. 2016), die auf technologiebasierte Innovationen und Entwicklungen zur ,Behebung des Anthropozäns setzen, greift Nut Brother zu einfachen Mitteln wie dem Staubsauger, um Luftverschmutzung zu dokumentieren. Nut Brother scheint dem fortschrittsorientierten Rahmen des ,guten' Anthropozäns ein wenig Wind aus den Segeln zu saugen beziehungsweise die Hoffnung auf Besserung und gegebenenfalls auch Bewältigung anthropogen verursachter Umweltkatastrophen etwas zu dämpfen.

Das ,gute‘ Anthropozän ähnelt dem Diskurs der Postpolitik. Die beiden Ansätze vereint, dass die Konflikthaftigkeit und Streitbarkeit von Lösungen anthropogener Umweltprobleme durch den Glauben an den erreichbaren „Erfolg' und die abschließende ,Lösung' anthropogener Probleme durch Technologie ausgehebelt werden. Das ,gute‘ Anthropozän sei durch smarte und flexible Technologien oder ein ,richtiges 'Verständnis von Wissenschaft, menschlicher Kreativität und Innovationsfähigkeit geprägt und zu etablieren, so die Verfechter*innen.[11] Ob Nut Brother nun diese Technologiegläubigkeit ad absurdum führen wollte oder nicht - sein unermüdlicher Streifzug durch Beijing als vereinzelter Spaziergänger*innen unterstreicht fast sentimental, dass ,wir ${ }^{\star}$ Menschen dem Anthropozän doch mehr ausgeliefert sind als jeder Glaube an technologische Innovation uns vermuten lässt.

Die Schwierigkeit der Sichtbarmachung von Smogbelastung wird von Nut Brother jedoch nicht nur visualisiert und physisch festgehalten, sondern auch performt: Nut Brothers persönliche Präsenz im Beijinger Stadtraum und der Einsatz des eigenen Körpers im öffentlichen Raum evozierte laut Nut Brothers Erzählungen teils ungeplante Irritationen und Nachfragen von Tourist*innen, lokalen Bewohner*innen und auch Sicherheitspersonal vor öffentlichen Gebäuden und Sehenswürdigkeiten. Diese temporäre körperliche Investition schätzt Nut Brother als notwendig für die politische Wiederaneignung des öffentlichen Raums ein: „Öffentliche Themen erfordern den öffentlichen Raum zur Diskussion, aber in China schrumpft der öffentliche Raum extrem [...]. Zurzeit hoffe ich, dass die Kunst ein öffentlicher Platz ist.“(NB 2019[12])

Somit sieht Nut Brother gerade in seinem ungefragten Auftreten beziehungsweise Eingriff in den öffentlichen Raum eine Möglichkeit, das Politische mit einem künstlerischen Akt zurückzubringen. Weiterhin erachtet Nut Brother eine gefühlsbetonte Ansprache und Einbeziehung des Publikums als wesentlich für die Wirksamkeit seiner Arbeiten: „Das Publikum soll seine Gedanken, Emotionen, Wünsche und Erinnerungen in diese Arbeit einfließen lassen, die mitschwingen und [die Arbeit] in 
Verlegenheit geraten lassen." (NB 2019) Das Affektive spielt also sowohl für die Produktion als auch Rezeption der Luft-Problematik eine Rolle. Das Publikum, welches im Kontext der Performance eher zufällig, ungeplant und vielleicht sogar ungewollt zum Publikum gemacht wird, erscheint ähnlich wie bei Amy Balkins Arbeit nicht so wichtig. Wichtiger erscheint, dass es eine Aktivierung und Bewusstseinsschaffung für die gefährlichen Auswirkungen von Smog gibt oder geben kann. Kurz gesagt, die Affizierung steht vor einem eindeutigen Effekt (im Sinne von Ziel) der Performance. Die Reaktionen oder Gefühle, die die künstlerische Aktion anstößt, bleiben letztlich außerhalb des Wirkungskreises des Künstlers selbst.

Entsprechend umfassten Reaktionen auf Nut Brothers Arbeit aus Lehm und Feinstaubpartikeln auch Spott und ironische wortwörtliche Übertragen der Stein-Metapher in den Alltag chinesischer Mitbürger*innen. Nut Brother berichtet:

„Die Leute sagten: ,Wow, jeden Tag. Es gibt Ziegelsteine zum Essen. Meine Mutter hat keine Angst mehr, dass ich Hunger habe.' Sie sagten: ,Ich kann mir nicht leisten, ein Haus zu kaufen; ich kann selbst Ziegelsteine dazu schaffen, um ein Haus zu bauen.' In Beijing gibt es Zehntausende von Ziegelsteinen." (NB 2019)

Das Angebot, den Smog-Backstein für 1.60o US-Dollar (ca. 1.40o Euro) zu verkaufen, lehnte Nut Brother mit der Begründung ab, das Ziel der Aktion sei nicht Profitgenerierung, sondern das Anstoßen eines Bewusstseinswandels. Generell machen weder der Backstein noch das performative Sammeln von Smogpartikeln allein die Kunst Nut Brothers aus. Und vielleicht ist das, was Nut Brother tut, auch gar keine Kunst, sondern eine politisch bewusst angewandte Ästhetik. Wie bei Balkin steht das vielgliedrige Zusammenspiel materieller, symbolischer, affektiver und auch kommunikativer Komponenten im Vordergrund; schließlich wurde Nut Brothers Aktion in einer Vielzahl internationaler Zeitungen, vom britischen Guardian (Phillips 2015) bis hin zur New York Times besprochen (Buckley/Wu 2015). Die Assemblage spricht auf mehreren Ebenen affektiv an, sie schafft Atmosphären eher als Objekte.

Nut Brother verortet sein Schaffen explizit als politisch, denn „in China ist es schwer zu sagen, welche Aspekte meiner Arbeit nicht politisch sind“ (NB 2019). Die Negativabgrenzung im Sinne von: ,Es ist politisch, weil es nicht nicht politisch ist', rekurriert auf ein umfassendes Verständnis des Politischen im Sinne der oben angesprochenen Gegner*innen des Postpolitischen. Darüber hinaus weist Nut Brother auf die scharfe Kriminalisierung vonseiten der chinesischen Regierung hin, die Kunst-Aktivist*innen mit „Terrorist*innen“ gleichsetze, was eine „sehr interessante Metapher“, aber auch „seltene Ehre“ zugleich sei (NB 2019). Offen spricht er von Luftverschmutzung als Problem ,für alle‘ und kritisiert die Verwehrung oder den Verlust des ,Rechts، auf frische Luft (NB 2019). Nut Brother macht das Ausmaß der anthropogenen ,Airpocalypse' (Phillips 2015) als konkrete Tatsache sichtbar, ,concrete bedeutet schließlich auch Beton im Englischen. Zudem kollektiviert Nut Brother, ähnlich wie Balkin, die Betroffenheit von anthropogener Luftverschmutzung durch die Ansprache eines nicht weiter differenzierten, betroffenen, wir'. Das eindrückliche Objekt des Backsteins vergegenwärtigt die Spuren des Menschen in der Stadt als harte, matter 
of fact ${ }^{\star}$, also als wissenschaftliche und evidenzbasierte Tatsache. Über die rein wissenschaftliche Dokumentation von Emissionslevels hinaus übermitteln Assemblage-Bausteine wie der Smog-Backstein jedoch zugleich ein affektives Potenzial als ,matter of concern', wie Erik Swyngedouw (2010: 218) in Anlehnung an Bruno Latour aufzeigt. Die oben anklingende Sorge über die Steine, die sich aus Smog bilden lassen und in unsere Lungen eindringen können, mobilisieren die Materialität des Smogs als Bollwerk gegen den postpolitischen Versuch, Klimawandel und Luftverschmutzung als unwiderrufbare Tatsachen zu zementieren. Anders gesagt, der Backstein als Manufakt verdeutlicht als Inbegriff menschengemachter Klimaverschmutzung gleichzeitig matters offact und matters of concern.

Nut Brothers Backstein entlarvt die anthropogenen Ursachen urbaner Luftverschmutzung - stellt sie als Ding dar, das da ist wegen ,uns ${ }^{\text {‘ }}$ - und (re-)aktiviert so die politische Verantwortung des Menschen für Umweltverschmutzung (Jasanoff 2010; Swyngedouw/Ernstson 2018). Im weiteren Sinne wirkt die Performance an der „Expansion und Pluralisierung kollektiver Verständnisse über die Desaster des Anthropozäns“ mit (Davis/ Todd 2017: 772, Übers. d. A.). Eingebettet in die lokalen und internationalen, spontanen und feuilletonistischen Reaktionen, Kritiken und Kommentare setzt Nut Brothers subtile Kritik an den Institutionen der Politik im engeren Sinne (chinesische Luftregulierungsbehörden, Regierungsinstitutionen und Industrieakteur*innen) beziehungsweise deren nicht ausreichendem Engagement gegen die menschengemachte Luftbelastung an. Die Kritik an ,der Politik dringt so fast lautlos aus dem Bereich des Politischen auch in die Sphäre der Politik hinein, indem sie die alleinige Regierung von Luft und Luftverschmutzung durch die Behörden in Frage stellt. Die Politik meistert die Krise nicht ausreichend, die Überbleibsel und Konsequenzen der nachlässigen technokratischen Verwaltung der Smog-Krise wiegen schließlich schwer in Nut Brothers Hand. Andererseits fordert Nut Brother die eigenständige Wirkmächtigkeit des Politisch-Künstlerischen ein. Wenn auch mit anderen Methoden als Balkin (welche bis auf die Ebene internationaler Gremien der Vereinten Nationen Aufmerksamkeit erregte), bringt Nut Brother mit den einfachsten Mitteln die Verschmutzung auf den Tisch. Jede*r Beijinger*in könnte es ihm mit geringem Aufwand gleichtun und ebenso steinharte Tatsachen schaffen. Dringt diese erschreckend-erweckende Realität durch, sodass der Backstein als Werkzeug ephemeren Widerstandes turbulente Emotionen wie Wut, Unbehagen, Ungläubigkeit, Panik, Angst und Selbstermächtigung hervorrufen kann, liegt es in den Händen jede*r Einzelnen, den Backstein im Kampf gegen das Anthropozän einzusetzen.

\section{Smog Free Project - das käufliche Anthropozän}

Das „Smog Free Project“[13] (2013-2017) des niederländischen Designers Daan Roosegaarde und seines Design-Studios will saubere Luft als attraktives Gut für nachhaltigkeitsbewusste Konsument*innen ansprechen. Als Teil dieses Vorhabens wurde 2013 zunächst der weltgrößte Luftreiniger, der sieben Meter hohe „Smog Free Tower“, als Pilotversion in Rotterdam installiert, welcher es den Menschen ermöglichen soll, „saubere Luft umsonst zu atmen“ (ebd.). Der Tower wird von Roosegaarde als „eine lokale Lösung für saubere 
Luft“ dargestellt (ebd.). Der Verweis auf das ,kostenlose' Nutzen sauberer Luft, die durch den „Smog Free Tower“ mithilfe eines Ionisierungsverfahrens bereitgestellt wird, bringt im Gegensatz zu den zwei vorherigen Projekten ein Verständnis von Luft als Ware zum Vorschein, wogegen sich insbesondere Amy Balkin explizit auflehnt. Als urbane Innovationen präsentiert, entstanden neben dem „Smog Free Tower“, der mittlerweile auch temporär in Polen und China aufgestellt worden ist, weitere „Smog-Free“-Produkte wie der „Smog Free Ring“beziehungsweise „Smog Free Manschetten“ und das „Smog Free Bike" mit einem integrierten Luftfilter, welches in Kooperation mit der größten chinesischen Bike-Sharing-Firma ofo angeboten wird. Roosegaardes Werk ist eine Assemblage der besonderen Art, schließlich kombiniert sie nicht nur diskursive und materielle Komponenten, sondern auch kommerzielle und öffentlich frei zugängliche Bestandteile miteinander. Roosegaardes thematische „Mission für saubere Luft“ (Schlick 2019) zieht sich durch die Produkt-Reihe sowie extern finanzierte Projekte wie den „Smog Free Tower“. Für jeden Geschmack soll etwas dabei sein.

Im Gegensatz zu Nut Brothers Backstein, der dank der unvorhergesehenen internationalen Aufmerksamkeit zum begehrten Objekt und zur potenziellen Ware aufstieg (und trotzdem nicht verkauft wurde), bilden bei Roosegaarde die städtischen Smogpartikel den nicht wegzudenkenden Hauptbestandteil für seine „Smog-Free“-Produktreihe. Der Verkaufspreis der Objekte von je 250 Euro (der „Smog-Free“-Ring wird auf der Website als potenzieller Verlobungsring angepriesen) soll in einem ,alternativen“ Emissionshandel im Gegenzug 10.000 Kubikmeter saubere Luft für die Allgemeinheit erkaufen (vgl. Abb. 4). Im Gegensatz zu Balkins Imitation oder Umwertung des Emissionshandels auf internationaler Ebene wendet Roosegaarde das ökonomische Prinzip des Kaufens und Verkaufens sauberer Luft auf sein eigenes gestalterisches Schaffen an und reproduziert oder erweitert gar die Kommodifizierung von Luft, wenn auch gegebenenfalls nicht so von ihm intendiert.

Weiterhin präsentiert Roosegaarde sein Werk als lokale „Lösung für das Problem anthropogener Luftverschmutzung" und es wird auch in Zeitschriften (Schlick 2019) und in Architektur- und Design-Diskursen als Lösungsbringer beschrieben.[15] Hier scheint Roosegaardes (implizite) Sympathie mit dem ,guten“ Anthropozän durch. Saubere Luft wird als erstrebenswertes Gut positioniert und mithilfe der, richtigen ' technologischen Innovationen aus

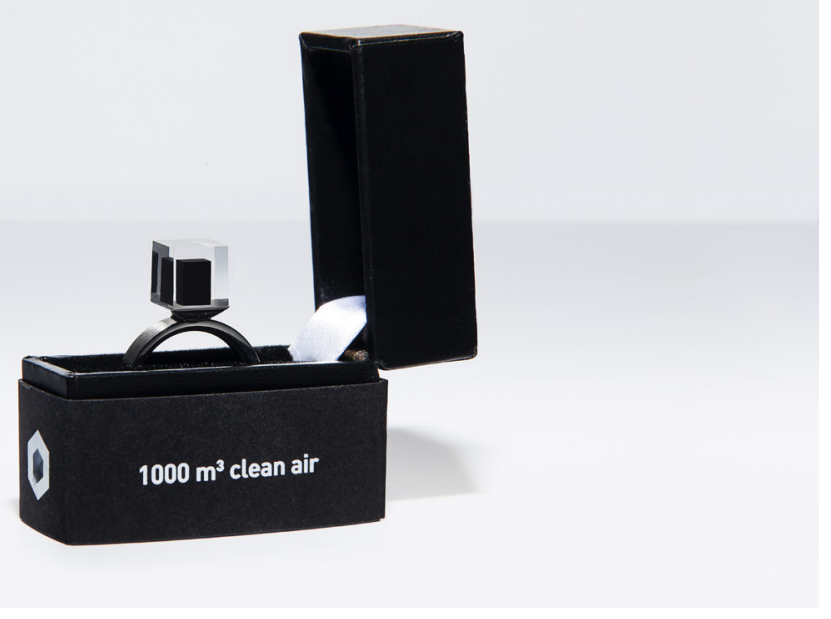

Abb. 4 „Smog-Free“Ring (Quelle: Studio Roosegaarde[14]) 
Roosegaardes Schmiede massenfähig verbreitbar und konsumierbar. Durch die Herstellung und den Vertrieb von „Smog-Free“-Produkten stellt Roosegaarde die Möglichkeit in Aussicht oder nährt den Glauben daran, dass das Anthropozän mittels technologischer Innovationen partiell behebbar sei. Gleichzeitig produziert dieses Denken jedoch auch eine Ko-Abhängigkeit zwischen der fortwährenden Verschmutzung und dem Erschaffen neuer Konsumprodukte.

Mit diesem techno-optimistischen Ansatz des ,guten“ Anthropozäns, der ressourcenoptimierende Verfahren als Lösung in den Vordergrund stellt, ergeben sich eigentlich neue politische Fragen und Herausforderungen, die verhandelt werden müssen. Im Sinne des postpolitischen Diskurses scheint Roosegaardes Ansatz jedoch die ,Lösungen“, die die „Smog-Free“-Produkte anbieten, eher als abschließende Lösung für das Problem einzuordnen, anstatt auf das eigentlich nicht abzuschließende Dilemma des Politischen beziehungsweise des Anthropozäns zu verweisen. Anders gesagt: Obwohl technologische Innovationen natürlich hilfreich zum Rückbau von Ressourcenzerstörung sein können, addieren sich all diese Anpassungs- oder Verbesserungsbemühungen gegen das Anthropozän nicht zu einem finalen Abschluss des Problems. Das Problem bleibt, und muss ebenso wie seine Lösungen stets neu verhandelt werden.

Während der Techno-Optimismus mancher Anthropozän-Denker*innen, die technologischen Fortschritt als ausreichende und heilbringende Auflösung für die mannigfaltigen Konsequenzen des Anthropozäns halten, depolitisierend wirken kann (Hamilton 2014; Rowan 2015), wären ein TechnoPessimismus oder andere Fundamentalkritiken gegen technologische (oder wissenschaftliche) Annäherungen zur Analyse anthropogener Umweltproblemlösung natürlich nicht minder gefährlich. Rory Rowan (2015) skizziert in diesem Zusammenhang überzeugend einen, linken Optimismus', der das immense Ausmaß des Anthropozäns mit einer politischen Auseinandersetzung über geo-soziale Zukünfte verknüpft. Dieser linke Optimismus könnte dazu anleiten oder affizieren, die notwendigerweise konfliktgeladenen Konturen jeglicher Technologien nicht aus den Augen zu verlieren.

Es bleibt fraglich, ob diese Einstellung bei Roosegaardes selbstsicherem Glauben, durch ,smarte' Produktentwicklungen anthropogenen Krisen Einhalt gebieten zu können, diesen linken Optimismus unterstützt. Problematisch an Roosegaardes Projekt ist somit nicht, dass Technologie als Komponente zur ,Behebung' des Anthropozäns betrachtet wird (wie auch immer diese dann aussehen würde - eine Welt voller „Smog Free Towers“?), sondern, dass die „Smog-Free“-Maßnahmen nicht als kontingent, umstritten und partiell, sondern als lösungsbringende Produkte im schlimmsten Fall die ,Mission für saubere Luft‘ depolitisieren. Roosegaardes begehrenswerte Produkte affizieren - sie sind schön, glatt, edel, gut für die Umwelt. Statt Unbehagen und vielleicht ein Gefühl von Machtlosigkeit gegen die globale Luftkrise zu empfinden, lehnt mensch sich gegebenenfalls nach dem Kauf der „Smog-Free“-Produkte mit Genugtuung zurück. Wer nicht wenig Geld für bessere Luft ausgegeben hat, hat vielleicht das Gefühl, den Dienst für die Allgemeinheit damit schon getan zu haben; und was Schönes für den privaten Lifestyle gab es noch mit dazu. Das schwächt die Suche nach Lösungen, die über das existierende kapitalistische System hinausgehen, eventuell ab 
(Neimanis et al. 2015; Swyngedouw 2010). Somit verspielt Roosegaarde nicht nur die Kritik am konsumorientierten und kommodifizierten Umgang mit Luft, sondern glättet dem techno-optimistischen, postpolitischen Regime mit Feel-good-Produkten das Gesicht.

\section{Ausblick: Das Politische im Anthropozän offenhalten}

Im Spannungsfeld des öffentlichen und privat(isiert)en Gutes ,guter ' Luft verweisen die Projekte verschiedentlich auf die radikale Interdependenz und gemeinsame Verantwortung für die Steuerung von Luft. Die vielseitigen Aufrufe und Affizierungen der aktivistischen Künstler*innen sprechen diffuse Publika als Betroffene, Mitverursacher*innen, Konsument*innen und mögliche zukünftige Allianzpartner*innen urbaner Luftverschmutzung an. Die Projekte zeigen nicht nur fantasievolle Strategien zum Umgang mit der Problematik von urbanem Smog auf, sondern aktivieren auch eine Vielzahl von Gefühlen, Gegenständen, Bildern, Gedanken und Politiken für die Aushandlung von Zukünften der sozialökologischen Transformation in verschmutzten Städten. Ästhetische und kreative Zugänge zu komplexen Problemen können in Ergänzung zu wissenschaftlichen Daten und Fakten affektives Wissen mobilisieren (Ingrim 2011). Wir haben gezeigt, dass die Projekte von divergierenden Grundannahmen über Luft als Gut ausgehen: Nut Brother und Amy Balkin sehen Luft eher als öffentliches, unteilbares Gut, Roosegaarde eher als zu verkaufende und zu verarbeitende Ressource.

Mit ihren künstlerisch-gestalterisch-performativen Praktiken und Produkten evozieren die Ansätze vielfältige affektive Reaktionen wie Angst, Depression, Entmutigung, Scham, Betroffenheit, Empathie, Wut, Sorge, Hoffnung, Demut bis hin zu Konsumentscheidungen oder politischen Aktionen. Mittels kreativer Aneignungen von bestehenden Institutionen und Normen der Politik entstehen neue Verbindungen zwischen Politik und dem Politischen (zum Beispiel ungebetene Kritik, neue Allianzen, Unterwanderung existierender Systeme, neue Warenkreisläufe durch/gegen Luftverschmutzung etc.). Umgehungs- und Anfechtungsstrategien, wie sie in Amy Balkins „Public Smog“ und Nut Brothers Performance „Project Dust“ deutlich werden, fordern die angebliche Omnipräsenz der Postpolitik heraus. Die ephemeren, temporären, zeit- und ressourcengebundenen Formen der politischen Mobilisierung gehen in ihrer Transmission von Affekten über eindeutige Bedeutungen und Intentionen hinaus (Kølvraa 2015) und machen doch deutlich, dass die affektiven Assemblagen materielle, diskursive, lokale und globale Aspekte des Anthropozäns miteinander in Zusammenhänge bringen. Das affektive Feedback, oder, feelback, welches diese affektiven Assemblagen durch Installationen, Objekte, Orte, gedankliche Projektionen, Protest-Aktionen, Wertgegenstände und spontane oder überlegte Äußerungen und Reaktionen potentiell hervorrufen (können), widerstrebt bewusst und anhaltend der Einordnung in gute oder schlechte Emotionen. Es schwingt vielmehr in affektiver Ambivalenz, was sich für wen gut oder schlecht anfühlt, sowie in der Ambivalenz zwischen Handeln und Fühlen.

Die drei vorgestellten mikropolitischen Interventionen individualisieren, kollektivieren, lokalisieren, globalisieren, materialisieren, diversifizieren, spekulieren, mobilisieren, kommodifizieren und kritisieren den Begriff und 
die Ära des Anthropozäns. Die Aktionen betreffen sowohl lokalspezifische Problemlagen urbaner Luftverschmutzung als auch skalenübergreifende Ebenen des Phänomens Luft; sie verknüpfen lokale und globale Probleme zu glokalen Kategorien von temporärer Sichtbarkeit, Sagbarkeit und Darstellbarkeit des Anthropozäns (Ruddick 2015: 1118). In der Neuordnung räumlicher und affektiver Register wird das Anthropozän in seiner Heterogenität und radikalen Interdependenz erfahr- und fühlbar.

In Rückkopplung an die Wechselwirkung zwischen Postpolitik und Anthropozän zeigen die Projekte unterschiedliche Umgangsweisen mit den Möglichkeiten politischer Macht, Gegenmacht, Ermächtigung oder Machtlosigkeit auf. Mag man beim Kaufvon Roosegaardes Produkten einen Moment von individuellem Erfolg oder temporärer Rückeroberung der eigenen Handlungsmacht verspüren und der Überzeugung sein, die Welt mit dem Kauf eines schönen Objektes ein bisschen besser gemacht zu haben, schwankt Balkin zwischen konkreten Handlungsaufforderungen und spekulativen Vorschlägen für bessere Luft. Nut Brother gibt keine Handlungsanleitung, was nach dem Erleben seiner Performance zu tun sei. Dieser nonverbale Appell kann jedoch gerade durch seine Offenheit vielleicht eine Vielzahl vorübergehender Antworten auf das Problem namens Anthropozän geben. Somit würde die Vormachtstellung von Verantwortlichen des angeblich alternativlosen Ist-Zustandes des Anthropozäns, die unter Umständen auch von der Nicht-Regulierung der Luftkrise profitieren, hinterfragt. Bleibt das Politische, zum Beispiel in den Formen von aktivistischer Selbstorganisation, Protesten, Allianzen dauerhaft und notwendigerweise umstritten, öffnet sich ein (Ver-)Handlungsraum, der eine politische Richtung oder einen Kurs eher erstreitet als einen Konsens. Die Projekte erinnern uns mit unterschiedlichen Mitteln daran, dass die Grenzen zwischen Natur, Kultur und Kommerz ebenso wie jene zwischen Mensch und nicht-menschlichen Akteur*innen konstruiert und kontingent sind. Sie alle sind sowohl Subjekte und Objekte von Aushandlungsprozessen, die nicht in Konsensmaschinen verfeuert oder ausgeschlossen werden sollten, sondern gerade in ihrer Konflikthaftigkeit Eingang ins Politische finden könnten. Durch das Offenbleiben der politischen Differenz zwischen Politik und dem Politischen würden die antagonistische Bedeutung und der folglich ebenfalls umstrittene Umgang mit dem öffentlichen Gut Luft erhalten bleiben - und das Politische wäre vielleicht doch (noch) nicht ausgestorben.

Die Bauhaus-Universität Weimar unterstützt die Publikation dieses Beitrags durch eine institutionelle Vereinbarung zur Finanzierung von Publikationsgebühren. 


\section{Endnoten}

[1] Der Ausruf des Anthropozäns im Jahr 2000 erhob den Menschen zu einer geologischen Instanz, die das Erdsystem nachhaltig verändert und Massenaussterben einschließlich menschlicher Kollateralschäden impliziert (Crutzen/Schwägerl 2011; Crutzen/ Stoermer 2000).

[2] https://www.hkw.de/de/programm/themen/das_anthropozaen_am_hkw/das_ anthropozaen_am_hkw_start.php (letzter Zugriff am 20.3.2020).

[3] https://theanthropocene.org/film/ (letzter Zugriff am 20.3.2020).

[4] https://www.nhm-wien.ac.at/dahinschmelzen (letzter Zugriff am 20.3.2020).

[5] Angesichts der multi-sensorischen Belastung/Erfahrung der Stadt (Howes/Classen 2014; Low/Kalekin-Fishman 2018) möchten wir über das latente Primat des Visuellen beziehungsweise über, visuelle Verschmutzung (z. B. in Form von aktiver und passiver Werbung durch Bildschirme, Poster, Graffiti, Einschränkungen von Sichtachsen durch Bebauung oder den Verlust der Nacht durch Beleuchtung im Vordergrund, vgl. Portella 2016) in der städtischen Wahrnehmung hinausgehen. Gerade weil auch in der Problematisierung des Anthropozäns viel auf visuelle Medien wie Fotographie oder Film zurückgegriffen wird (Mirzoeff 2014; Nixon 2014) und damit Anthropozän als vorrangig visuelles Phänomen verstärkt werden könnte, fokussiert sich unsere Auswahl künstlerischer und gestalterischer Positionen auf installative, performative und objektbasierte Arbeiten, ohne jedoch die damit einhergehende Visualität der Projekte zu missachten. Weiterhin begrenzt sich die Fallauswahl auf künstlerisch-gestalterische Positionen, die sich spezifisch mit urbaner Luft, Luftverschmutzung, Staub und Smog beschäftigen.

[6] In der andauernden Kontroverse über die Wechselwirkungen und Unterschiede zwischen Affekt und Emotion, insbesondere im Hinblick auf Affekt als eingeschrieben in Körperlichkeit und/oder Materialität, sowie die Debatte, ob Affekt prä-kognitiv zu betrachten sei, verweisen wir auf die Schwierigkeit, Affekte ohne individuelle emotionale und verkörperlichte Wahrnehmungen analytisch zu erschließen (Hemmings 2005; Leys 2011). Wir fokussieren uns also auf die Mobilisierung von Affekten im weiteren Sinne als „Agent*innen für sozialen Wandel“ (Fox/Alldred 2017: 13), ohne diese streng nach Affekt oder Emotion zu unterscheiden (siehe auch Ahmed 2014: 97; Hemmings 2005; Wetherell 2012). Weiterhin nehmen wir keine Wertung oder Einordnung von affektiven Implikationen als gerichtet, wie zum Beispiel positiv oder negativ, gut oder schlecht, vor.

[7] In Bezug auf affektive Methodologien verstehen wir empirisches Material als, verkörperlichte Daten' (embodied data), die entweder ,emisch“ vom affizierten Körper selbst oder ,etisch von einer externen Beobachter*in produziert werden (Knudsen/Stage 2015: 8). Angesichts unserer Nicht-Teilnahme beziehungsweise unseres Nicht-Erlebens der Projekte folgt unsere Analyse ,etisch'. Das heißt, unsere eigenen affektiven Wahrnehmungen der künstlerischen Interventionen koproduzieren diese als affektive Assemblagen; die Assemblagen selbst gehen über singuläre oder materiell einzugrenzende Kunstobjekte hinaus. Die Datenauswertung umfasst Interviews mit Amy Balkin (AB) und Nut Brother (NB) sowie Dokumentationen der Performances, Presse- und Theorietexte. Eine Interviewanfrage an Daan Roosegaarde wurde höflich abgelehnt mit der Begründung, dass es sich um ein abgeschlossenes Projekt handele und der Fokus derzeit auf aktuellen Projekten liege.

[8] http://www.publicsmog.org/ (letzter Zugriff am 20.3.2020).

[9] Amy Balkin, online Interview, Berlin, 2.1.2019.

[10] http://twentynine.fibreculturejournal.org/fcj-219-the-sensed-smog-smart-ubiquitouscities-and-the-sensorial-body/ (letzter Zugriff am 20.12.2018).

[11] https://goodanthropocenes.net/definitions-of-a-good-anthropocene/ (letzter Zugriff am 23.3.2020).

[12] Nut Brother, online Interview, Berlin, 7.2.2019. Alle folgenden Zitate von Nut Brother stammen aus diesem Interview, im Folgenden gekennzeichnet als NB 2019.

[13] https://www.studioroosegaarde.net/project/smog-free-tower (letzter Zugriff am 24.3.2020).

[14] https://www.studioroosegaarde.net/project/smog-free-ring (letzter Zugriff am 23.3.2020).

[15] https://awards.mediaarchitecture.org/mab18/projects/ (letzter Zugriff am 23.3.2020). 


\section{Autor_innen}

Friederike Landau, Stadtsoziologin und politische Theoretikerin, interessiert sich für Formen künstlerischen Aktivismus im Spannungsfeld zwischen Politik und dem Politischen. friederike.landau@metropolitanstudies.de

Alexandra Toland, bildende Künstlerin und Landschaftsplanerin, arbeitet zu Dialogen zwischen Bodenwissenschaftler*innen und Künstler*innen sowie in ihrer eigenen künstlerischen Arbeit mit Staub.

alexandra.toland@uni-weimar.de

\section{Literatur}

Ahmed, Sara (2014): Affect/Emotion: Orientation Matters. A conversation with Sigrid Schmitz and Sara Ahmed. In: Freiburger Zeitschrift für GeschlechterStudien 20/2, 97-108.

Anderson, Ben (2009): Affective atmospheres. In: Emotion, Space and Society 2/2, 77-81.

Balkin, Amy (2012): Public Smog (2004-ongoing). In: Tecnoscienza - Italian Journal of Science and Technology Studies 2/3, 1-4.

Balkin, Amy (2015): Public Smog - A project by Amy Balkin. In: Etienne Turpin / Heather Davis Heather (Hg.), Art in the Anthropocene: Encounters Among Aesthetics, Politics, Environments and Epistemologies. London: Open Humanities Press, 341-347.

Bennett, Elena M. et al. (2016): Bright spots. Seeds of a good Anthropocene. In: Frontiers in Ecology and the Environment 14/8, 441-448.

Berke, Jeremy (2015): Beijing's air pollution is so bad a man made a ,smog brick' out of it. In: Business Insider, 1.12.2015. https://www.businessinsider.com/beijing-airpollution-inspires-man-to-make-a-smog-brick-2015-12?r=DE\&IR=T (letzter Zugriff am 23.3.2020).

Bignall, Simone (2010): Affect and Assemblage: Ethics beyond Enjoyment. In: Simone Bignall / Paul Patton (Hg.), Deleuze and the Postcolonial. Edinburgh: Edinburgh University Press, 78-102.

Bishop, Claire (Hg.) (2010): Participation. London: Whitechapel.

Buckley, Chris; Wu, Adam (2015): Amid Smog Wave, an Artist Molds a Potent Symbol of Beijing's Pollution.In:NewYorkTimes, 1.12.2015.https://www.nytimes.com/2015/12/02/ world/asia/beijing-smog-air-pollution-artist-brick.html (letzter Zugriff am 20.3.2020).

Buser, Michael / Bonura, Carlo / Fannin, Maria / Boyer, Kate (2013): Cultural activism and the politics of place-making. In: City 17/5, 606-627.

De Cauter, Lieven / de Roo, Ruben / Vanhaesebrouck, Karel (2011): Art and activism in the age of globalization. New York: NAi Publishing.

Crutzen, Paul / Schwägerl, Christian (2011): Living in the Anthropocene: Toward a New Global Ethos. In: Yale Environment 360, 24.1.2011. https://e360.yale.edu/features/ living_in_the_anthropocene_toward_a_new_global_ethos (letzter Zugriff am 20.3.2020).

Crutzen, Paul / Stoermer, Eugene (2000): The „Anthropocene.“ In: Global Change Newsletter 41, 17-18.

Davis, Heather / Todd, Zoe (2017): On the Importance of a Date, or, Decolonizing the Anthropocene. In: Critical Geographies 16/4, 761-780.

Davis, Heather / Turpin, Etienne (2015): Art in the Anthropocene: Encounters Among Aesthetics, Politics, Environments and Epistemologies. o. O.: Open Humanities Press.

Davis, Mike (1999): Ecology of Fear. Los Angeles and the Imagination of Disaster. New York: Vintage Books.

Demos, Thomas J. (2009): The Politics of Sustainability: Contemporary Art and Ecology. In: Barbican Art Gallery (Hg.), Radical Nature. Art and Architecture for a Changing Planet 1969-2009. Köln: Walther König, 16-30.

Demos, Thomas J. (2017): Against the anthropocene. Visual Culture and Environment Today. Santa Monica: Sternberg Press.

Derickson, Kate Driscoll / MacKinnon, Danny (2015): Toward an Interim Politics of Resourcefulness for the Anthropocene. In: Annals of the Association of American Geographers 105/2, 304-312. 
Estok, Simon C. / Chou, Shiuhhuah Serena (2017): The City and the Anthropocene. In: Concentric: Literary and Cultural Studies 43/1, 3-11.

Falb, Daniel (2015): Epistemologies of Art in the Anthropocene. In: Christoph Behnke / Cornelia Kastelan / Valérie Knoll (Hg.), Art in the Periphery of the Center. Berlin: Sternberg Press, 302-317.

Fox, Nicholas J. / Alldred, Pam (2017): Sociology and the New Materialism. Theory, Research, Action. Los Angeles u. a.: Sage.

Gregg, Melissa / Seigworth, Gregory J. (2011): The affect theory reader. North Carolina: Duke University Press.

Hamilton, Clive (2014): The New Environmentalism Will Lead Us to Disaster. So-called ecopragmatists say we can have a „good Anthropocene“. They're dead wrong. In: Scientific American 19.6.2014. https://www.scientificamerican.com/article/the-newenvironmentalism-will-lead-us-to-disaster (letzter Zugriff am 20.3.2020).

Haraway, Donna Jeanne (2016): Staying with the Trouble. Making Kin in the Chthulucene. (Experimental Futures: Technological Lives, Scientific Arts, Anthropological Voices.) Durham: Duke University Press.

Haraway, Donna Jeanne / Kenney, Martha (2015): Anthropocene, Capitalocene, Chthulhocene. In: Etienne Turpin / Heather Davis (Hg.), Art in the Anthropocene: Encounters Among Aesthetics, Politics, Environments and Epistemologies. London: Open Humanities Press, 255-270.

Hemmings, Clare (2005): Invoking Affect. In: Cultural Studies 19/5, 548-567.

Hieber, Lutz / Möbius, Stephan (2011): Ästhetisierung des Sozialen. Reklame, Kunst und Politik im Zeitalter visueller Medien. München: Beck.

Ho, Victoria (2015): Chinese artist creates a brick from Beijing's smog that he vacuumed. In: Mashable, 1.12.2015. https://mashable.com/2015/12/o1/beijing-smogbrick/?europe=true (letzter Zugriff am 23.3.2020).

Hollands, Robert / Vail, John (2012): The art of social movement. Cultural opportunity, mobilisation, and framing in the early formation of the Amber Collective. In: Poetics 40/1, 22-43.

Howes, David / Classen, Constance (2014): Ways of Sensing. Understanding the senses in society. London: Routledge.

Ingrim, Mrill (2011): Eliciting a response through art. In: Nature Climate Change 1, 133-134.

Jasanoff, Sheila (2010): A New Climate for Society. In: Theory, Culture \& Society 27/2-3, 233-253.

Jessop, Bob (2014): Repoliticising depoliticisation. Theoretical preliminaries on some responses to the American fiscal and Eurozone debt crises. In: policy \& politics 42/2, 207-223.

Juris, Jeffrey S. / Khasnabish, Alex (2013): Insurgent Encounters. Transnational Activism, Ethnography, and the Political. Durham/London: Duke University Press.

Kaika, Maria / Swyngedouw, Erik (2014): Radical urban political-ecological imaginaries Planetary urbanization and politicizing nature. In: Eurozine 2014.

Karaliotas, Lazaros / Bettini, Giovanni (2016): Urban resilience, the local and the politics of the Anthropocene: reflections on the future of the urban environment. In: Kevin Archer / Kris Bezdecny (Hg.), Handbook of Cities and the Environment. Northhampton: Edward Elgar Publishing, 1-17.

Kester, Grant H. (Hg.) (1998): Art, Activism, and Oppositionality. Essays from Afterimage. Durham: Duke University Press.

Klingan, Katrin / Sepahvand, Ashkan / Rosol, Christoph / Scherer, Bernd M. (Hg.) (2015): Textures of the Anthropocene. Grain, Vapor, Ray. Berlin: Haus der Kulturen der Welt.

Klöckner, Christian / Hertwich, Edgar / Borner, Jochen / Zienert, Martina / Kintisch, Eli / Jury, Sam / Buckham, D. / Swimm, J. / Stern, P.C. / Huybers, Peter (2014): Klimakunst als Kommunikationsmittel? CLIMART - Ein Projekt, das Potenziale und Grenzen auslotet. In: Kolleg für Management und Gestaltung nachhaltiger Entwicklung. http://kmgne. de/wp-content/uploads/2014/10/2014_Climart_Klimakunst.pdf (letzter Zugriff am 20.3.2020).

Knudsen, Britta Timm / Stage, Carsten (Hg.) (2015): Affective Methodologies. Developing Cultural Research Strategies for the Study of Affect. London: Palgrave Macmillan.

Kølvraa, Christoffer (2015): Affect, Provocation, and Far Right Rhetoric. In: Britta Timm Knudsen / Carsten Stage (Hg.), Affective Methodologies. London: Palgrave Macmillan, 183-201. 
Landau, Friederike (2019): Agonistic Articulations in the ,Creative‘ City. On New Actors and Activism in Berlin's Cultural Politics. London/New York: Routledge.

Landau, Friederike / Roskamm, Nikolai (2018): [Un]settling the City. In: engagee - Rebel Cities, Radical Democracy 6/7, 73-77.

Landau, Friederike / Toland, Alexandra (im Ersch.): Sensing the Injustice of Dust: Exploring Activist Artistic Approaches to Politicize Air Pollution.

Leys, Ruth (2011): The Turn to Affect. A Critique. In: Critical Inquiry 37/3, 434-472.

Low, Kelvin E. Y. / Kalekin-Fishman, Devorah (Hg.) (2018): Senses in Cities. Experiences of Urban Settings. London/New York: Routledge.

Marchart, Oliver (2010): Die politische Differenz: Zum Denken des Politischen bei Nancy, Lefort, Badiou, Laclau und Agamben. Berlin: Suhrkamp.

Marchart, Oliver (2013): Das unmögliche Objekt: Eine postfundamentalistische Theorie der Gesellschaft. Berlin: Suhrkamp.

McKee, Yates (2017): Strike Art. Contemporary Art and the Post-Occupy Condition. London/ New York: Verso.

Michel, Boris / Roskamm, Nikolai (2013): Einführung - Die ,postpolitische Stadt‘. In: sub \urban 1/2, 9-16.

Mirzoeff, Nicholas (2014): Visualizing the Anthropocene. In: Public Culture 26/2, 213-232.

Mohr, Henning / Landau, Friederike (2017): Interventionen als kreative Praxisform: Die Suche nach Neuheit als gesellschaftliches Phänomen, In: Julia-Lena Reinermann / Friederike Behr (Hg.), Experimentalstadt. Wiesbaden: Springer VS, 59-79.

Moore, Jason W. (2016): Anthropocene or Capitalocene? Nature, History, and the Crisis of Capitalism. St. Paul: KAIROS Press.

Mouffe, Chantal (2005): On the political (Thinking in Action). London/New York: Routledge.

Neimanis, Astrida / Åsberg, Cecili / Hedrén, Johan (2015): Four Problems, Four Directions for Environmental Humanities: Toward Critical Posthumanities for the Anthropocene. In: Ethics and the Environment 20/1, 67-97.

Nixon, Rob (2014): The Great Acceleration and the Great Divergence: Vulnerability in the Anthropocene. In: MLA Professional, März 2014. https://profession.mla.org/the-greatacceleration-and-the-great-divergence-vulnerability-in-the-anthropocene (letzter Zugriff am 20.3.2020).

Phillips, Tom (2015): China's vacuum-cleaner artist turning Beijing's smog into bricks. In: The Guardian, 1.12.2015. https://www.theguardian.com/world/2015/dec/o1/chinesevacuum-cleaner-artist-turning-beijings-smog-into-bricks (letzter Zugriff am 20.3.2020).

Portella, Adriana (2016): Visual Pollution. Advertising, Signage and Environmental Quality. London/New York: Routledge.

Reestorff, Camilla M. (2015): From Artwork to Net-Work: Affective Effects of Political Art. In: Britta Timm Knudsen / Carsten Stage (Hg.), Affective Methodologies. London: Palgrave Macmillan, 201-222.

Renn, Jürgen / Scherer, Bernd (Hg.) (2017): Das Anthropozän. Zum Stand der Dinge. Berlin: Matthes \& Seitz.

Renn, Jürgen / Karkowsky, Stephan (2018): Ein Massensterben, das der Mensch zu verantworten hat. In: Deutschlandfunk Kultur, 23.7.2018. https://www. deutschlandfunkkultur.de/anthropozaen-und-biodiversitaet-ein-massensterbendas-der.1008.de.html?dram:article_id=423565 (letzter Zugriff am 20.3.2020).

Rowan, Rory (2015): Extinction as Usual? Geo-Social Futures and Left Optimism. In: e-flux, 31.7.2015. http://supercommunity.e-flux.com/texts/extinction-as-usual-geo-socialfutures-and-left-optimism (letzter Zugriff am 20.3.2020).

Ruddick, Sue (2015): Situating the Anthropocene. Planetary urbanization and the anthropological machine. In: Urban Geography 36/8, 1113-1130.

Schlick, Leonie (2019): Großes Problem, smarte Lösung: Smog Free Tower. In: Capital, 8.7.2019. https://www.capital.de/wirtschaft-politik/grosses-problem-smarte-loesungsmog-free-tower (letzter Zugriff am 20.3.2020).

Scott, Emily Eliza (2014): Field Effects: Invisible-5’s Illumination of Peripheral Geographies. In: Art Journal 69/4, 38-47.

Street, Alice (2012): Affective Infrastructure. In: Space and Culture 15/1, 44-56.

Swyngedouw, Erik (2009): The Antinomies of the Postpolitical City. In Search of a Democratic Politics of Environmental Production. In: International Journal of Urban and Regional Research 33/3, 601-620. 
Swyngedouw, Erik (2010): Apocalypse Forever? In: Theory, Culture \& Society 27/2-3, 213-232.

Swyngedouw, Erik (2011): Interrogating post-democratization. Reclaiming egalitarian political spaces. In: Political Geography 30/7, 370-380.

Swyngedouw, Erik / Ernstson, Henrik (2018): Interrupting the Anthropo-obScene: Immuno-biopolitics and Depoliticizing Ontologies in the Anthropocene. In: Theory, Culture \& Society 35/6, 3-30.

Wetherell, Margaret (2012): Affect and Emotion. A New Social Science Understanding. London u. a.: Sage.

Woddis, Jane (2005): Spear-Carriers or Speaking Parts? Arts Practitioners in the Cultural Policy Process. Warwick: University of Warwick, Centre for Cultural Policy Studies.

Yusoff, Kathryn / Gabrys, Jennifer (2011): Climate change and the imagination. In: Advanced Review 2, 516-536.

\section{Seeing, smelling, saving air. The Anthropocene of the (post)political city}

This article discusses the post-political city within an increasing academic and activist debate about the Anthropocene, which describes the ecological and socio-political implications of human action on the earth's surface. Based on three empirical case studies, we explore how the specifically anthropogenic (i.e., human-made) crisis of urban air pollution is problematized in artistic projects. In the context of the acclaimed rise of postpolitics, we discuss, on the one hand, how the ambivalent discourse of the Anthropocene era favors depoliticization, and, on the other hand, how it opens up new possibilities for the repoliticization of global environmental challenges. 